\title{
DESIDRATAÇÃO DE SEMENTES DE SOJA EM LEITO FIXO: MODELAGEM E SIMULAÇÃO; ANÁLISE DE EFEITO DAS VARIÁVEIS E HETEROGENEIDADE DO PROCESSO
}

\author{
G. F. M. V e SOUZA ${ }^{1}$, R. F. de MIRANDA ${ }^{2}$, F. S. LOBATO ${ }^{1}$, M. A. S. BARROZO ${ }^{1}$ \\ ${ }^{1}$ Universidade Federal de Uberlândia, Faculdade de Engenharia Química \\ ${ }^{2}$ Universidade Federal de Uberlândia, Faculdade de Engenharia Mecânica \\ E-mail para contato: glaucia.souza@gmail.com
}

\begin{abstract}
RESUMO - O objetivo deste trabalho foi realizar um estudo do processo de secagem de sementes de soja em leito fixo e camada espessa. Foi realizada a simulação matemática deste processo utilizando um modelo a duas fases, com a utilização do método das linhas para resolução numérica. Os dados experimentais dos perfis de umidade e temperatura das sementes de soja e da temperatura do ar de secagem em diferentes posições axiais do leito fixo no decorrer do tempo foram comparados com os valores simulados, mostrando uma boa concordância, com desvios médios variando de 1,4 a 5,7\%. Quando a secagem é realizada em leito fixo existem diferenças significativas de umidade, de temperatura do ar e das sementes na direção axial, sendo que, como esperado, as menores umidades das sementes e maiores temperaturas do ar e das sementes foram alcançadas nas posições mais próximas da entrada do ar no leito $(0,05$ e $0,10 \mathrm{~m})$. De modo geral, a taxa de secagem cresce com o aumento da temperatura e da velocidade, juntamente com a diminuição da umidade relativa do ar de secagem. Em apenas quatro experimentos as sementes obtiveram a umidade final abaixo ou próximas de 13 a 14\% (bs) nas diferentes posições axiais do leito e que apenas um apresentou as condições menos desfavoráveis para a qualidade das sementes $\left(\mathrm{T}=40^{\circ} \mathrm{C} ; \mathrm{V}=0,7 \mathrm{~m} / \mathrm{s} ; \mathrm{UR}=35 \%\right)$.
\end{abstract}

\section{INTRODUÇÃO}

A secagem artificial da semente de soja envolve uma série de particularidades. A literatura traz relatos sobre alterações na qualidade das sementes durante a secagem, seja em leitos móveis ou leitos fixos (Lisboa et al, 2007; Barrozo et al, 1996). Devido ao cuidado que deve ser tomado em qualquer sistema que envolve mover as sementes, o que pode levar a danos mecânicos, é recomendado o uso de secadores de leito fixo. Neste tipo de secador, a semente permanece estática e o ar de secagem é forçado a passar através do espaço intersticial da massa de sementes até que sua umidade atinja em torno de $14 \%$ (base seca - bs), o que proporciona um armazenamento seguro sem grandes prejuízos à qualidade das sementes. 
A conveniente modelagem e simulação computacional dos perfis de temperatura e umidade no secador permite, por exemplo, examinar e interpretar a influência das condições operacionais sobre o processo sem a necessidade de recorrer a um extensivo conjunto de testes experimentais. Além disto, uma maior compreensão da transferência de calor e massa entre o sólido e o fluido contribui para o projeto e controle de novos secadores, bem como para a otimização de secadores já existentes, assegurando a obtenção de um produto final em condições adequadas de beneficiamento e armazenamento (López et al, 1998). Contudo, deve ser ressaltado que a experimentação é de fundamental importância dentro de qualquer pesquisa sobre secagem, uma vez que pode contribuir para a compreensão da física do processo, para o desenvolvimento de modelos mais realísticos e para a verificação da precisão e credibilidade das simulações.

O objetivo deste trabalho foi fazer um estudo detalhado do processo de secagem de sementes de soja em leito fixo, considerando a modelagem e simulação do processo, em conjunto com um estudo experimental de verificação e heterogeneidade do processo, através das variáveis de secagem, além de uma análise de efeito dessas variáveis na qualidade final da semente.

\section{MATERIAL E MÉTODOS}

\subsection{Material e Aparato experimental}

Para os procedimentos experimentais foram utilizadas sementes comerciais de soja da variedade BRS Valiosa, geneticamente modificada, com diâmetro médio de Sauter $\mathrm{dp}=6 \times 10^{-3} \mathrm{~m}$, densidade $\rho=1,19 \times 10^{-3} \mathrm{~kg} \cdot \mathrm{m}^{-3}$, e com o leito apresentando porosidade $\varepsilon=0,37$.

A Figura 1 descreve a unidade piloto experimental utilizada neste trabalho.

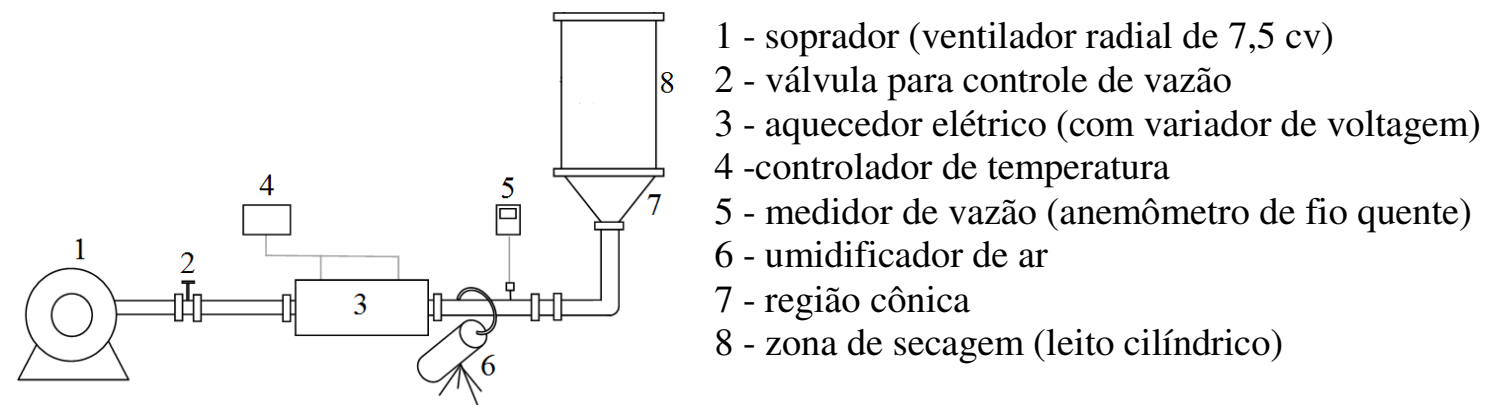

Figura 1. Esquema da unidade piloto experimental

\subsection{Condições operacionais}

Foram 16 condições operacionais, determinadas através de um planejamento composto central (Box et al, 1978), considerando duas repetições no ponto central. A Tabela 1 mostra os 
níveis de temperatura $\left(\mathrm{T}_{f}\right)$, velocidade superficial $\left(\mathrm{V}_{f}\right)$ e umidade relativa $\left(\mathrm{UR}_{f}\right)$ do ar de secagem utilizadas neste trabalho.

Tabela 1. Níves das condições operacionais

\begin{tabular}{cccc}
\hline Níveis & \multicolumn{3}{c}{ Variáveis } \\
\hline$x$ & $\mathrm{~T}_{f}\left({ }^{\circ} \mathrm{C}\right)$ & $\mathrm{V}_{f}(\mathrm{~m} / \mathrm{s})$ & $\mathrm{UR}_{f}(\%)$ \\
$-\alpha=-1,41$ & 32,9 & 0,27 & 20,8 \\
-1 & 35,0 & 0,4 & 25,0 \\
0 & 40,0 & 0,7 & 35,0 \\
+1 & 45,0 & 1,0 & 45,0 \\
$+\alpha=1,41$ & 47,0 & 1,12 & 49,1 \\
\hline
\end{tabular}

A precisão das medidas para os valores de temperatura, velocidade e umidade relativa do ar de secagem foram de cerca de $\pm 0.3^{\circ} \mathrm{C}, \pm 0,05 \mathrm{~m} / \mathrm{s}$ e $\pm 4 \%$ respectivamente.

\subsection{Modelo matemático}

Algumas considerações são assumidas para o desenvolvimento do modelo (Brooker et al, 1974): os escoamentos do fluido tem perfil plano de velocidade; a convecção na superfície do sólido é o mecanismo predominante no processo de troca térmica; perda de calor é desprezível através das paredes do sistema; transferência de calor na direção normal aos escoamentos é desprezível em relação à transferência de calor nas direções dos escoamentos; leito com propriedades homogêneas; escoamentos unidirecionais do fluido e as propriedades físicoquímicas constante ao longo do leito.

O balanço de energia e de massa é feito através da análise das trocas em um volume diferencial localizado no leito fixo de secagem, gerando as equações apresentadas na Tabela 2 , juntamente com as condições iniciais de alimentação de umidade $(W)$ e temperatura do ar $\left(T_{f}\right)$ adotadas e de umidade $(M)$ e temperatura do sólido $\left(T_{s}\right)$ (Brooker et al, 1974).

Para prever o teor de umidade de equilíbrio $\left(\mathrm{M}_{\mathrm{eq}}\right)$ de sementes de soja foi utilizada a equação Halsey modificada (Osborn et al., 1989), com parâmetros estimados por Barrozo (1996). O mesmo autor obteve a equação de cinética de secagem de sementes de soja, a partir de dados de secagem em camada fina. Com relação ao coeficiente de transferência de calor (h) a correlação utilizada para leito fixo proposta por Wakao, Kaguei e Funazkri (1979) já foi utilizada com sucesso para grãos de soja, sendo utilizada também no presente trabalho. 
Tabela 2. Equações do modelo a duas fases e condições iniciais de umidade e temperatura do sólido e de alimentação de umidade e temperatura do ar adotadas

\begin{tabular}{|c|c|c|}
\hline \multirow{2}{*}{$\begin{array}{c}\text { Conservação } \\
\text { de massa }\end{array}$} & Fluido & $\frac{\partial W}{\partial t}=\frac{1}{\varepsilon \rho_{s}}\left(f-G_{f} \frac{\partial W}{\partial \mathrm{y}}\right)$ \\
\cline { 2 - 4 } & Sólido & $\frac{\partial M}{\partial t}=\frac{-f}{(1-\varepsilon)}$ \\
\hline \multirow{2}{*}{$\begin{array}{c}\text { Conservação } \\
\text { de energia }\end{array}$} & Fluido & $\frac{\partial T_{f}}{\partial t}=\frac{-h A\left(T_{f}-T_{S}\right)-G_{f}\left(C p_{f}+W C p_{v}\right) \frac{\partial T_{f}}{\partial t}}{\varepsilon \rho_{f}\left(C p_{f}+W C p v\right)}$ \\
\cline { 2 - 4 } & Sólido & $\frac{\partial T_{s}}{\partial t}=\frac{h A\left(T_{f}-T_{s}\right)-f\left(\lambda+C p_{v}\left(T_{f}-T_{S}\right)\right)}{(1-\varepsilon) \rho_{S}\left(C p_{S}+\rho_{S} M C p_{l}\right)}$ \\
\hline $\begin{array}{c}\text { Condições de } \\
\text { contorno }\end{array}$ & Fluido & $\begin{array}{l}W=W(0, \mathrm{t})=W_{0} \\
T_{f}=T_{f}(0, \mathrm{t})=T_{f 0}\end{array}$ \\
\hline $\begin{array}{c}\text { Condições } \\
\text { iniciais }\end{array}$ & Sólido & $\begin{array}{l}M=M(\mathrm{y}, 0)=M_{0} \\
T_{s}=T_{s}(\mathrm{y}, 0)=T_{s 0}\end{array}$ \\
\hline
\end{tabular}

Para resolução do modelo proposto foi utilizado o método das linhas. Foi utilizado o software Matlab ${ }^{\circledR}$ para resolver as derivadas temporais.

\subsection{Metodologia experimental}

As sementes de soja foram reumidificadas artificialmente por meio da absorção do vapor de água saturado (Barrozo et al, 1998) pela semente até atingirem a umidade inicial desejada para os experimentos, em torno de $20 \pm 1 \%$ (bs). Após a reumidificação foi retirada uma amostra de sementes para avaliação da sua qualidade inicial.

As sementes de soja eram colocadas no leito fixo formando uma camada espessa de $0.4 \mathrm{~m}$, iniciando a contagem de tempo do experimento (tempo zero). Foram retiradas amostras de sementes aos 20, 40, 70, 110, 140 e 180 minutos para medida de umidade pelo método da estufa $\left(105 \pm 3{ }^{\circ} \mathrm{C}\right.$ por 24 horas), medida de temperatura das sementes e do ar aos $25,55,85,120,160$ e 180 minutos. Para as medições de temperatura das sementes, as amostras foram imediatamente colocadas em pequenos recipientes isolados termicamente e um termopar de cobre-constantan, instalado no interior de cada recipiente efetuou a medida.

Para análise da qualidade das sementes de soja, ao final dos 180 minutos, foram retiradas amostras na direção axial do leito para realização dos testes de germinação e o de sementes sem fissuras, conforme Souza (2012). Os valores obtidos foram divididos pelos respectivos valores da amostra retirada após a reumidificação (qualidade inicial, antes da secagem), obtendo-se assim o índice de germinação (IG) e o índice sementes sem fissuras (ISSF). Visando analisar os efeitos da 
três variáveis do processo de secagem, temperatura, velocidade superficial e umidade relativa do ar de secagem, e suas interações, na qualidade das sementes, foi empregada a metodologia estatística utilizando a técnica de superfície de resposta (Myers; Montgomery, 2002).

As amostragens foram efetuadas na direção axial do leito nas posições: $0,05,0,10,0,20$, 0,30 e $0,40 \mathrm{~m}$.

\section{RESULTADOS}

A Figura 2 mostra resultados típicos para os perfis de umidade da soja (bs), temperatura das sementes $\left({ }^{\circ} \mathrm{C}\right)$ e temperatura do ar de secagem $\left({ }^{\circ} \mathrm{C}\right)$, respectivamente, para os dados experimentais (exp) e as respectivas respostas simuladas (sim). O comportamento destes resultados foi observado também nas demais situações estudadas. De um modo geral, o modelo a duas fases forneceu bons resultados para representar a transferência de calor e massa do processo de secagem de sementes de soja em leito fixo. Considerando que não foram feitos ajustes do modelo aos dados experimentais e sim a comparação dos dados experimentais com os resultados de umidade das sementes previstos pela simulação, com os parâmetros originais das equações constitutivas, considerando ainda a incerteza experimental dessas medidas, pode se dizer que a simulação previu adequadamente a variação da umidade e da temperatura das sementes e da temperatura do ar na direção axial do secador em leito fixo, com desvios médios de 5,7; 5,5 e 1,4 $\%$, respectivamente (Figura 2 ).
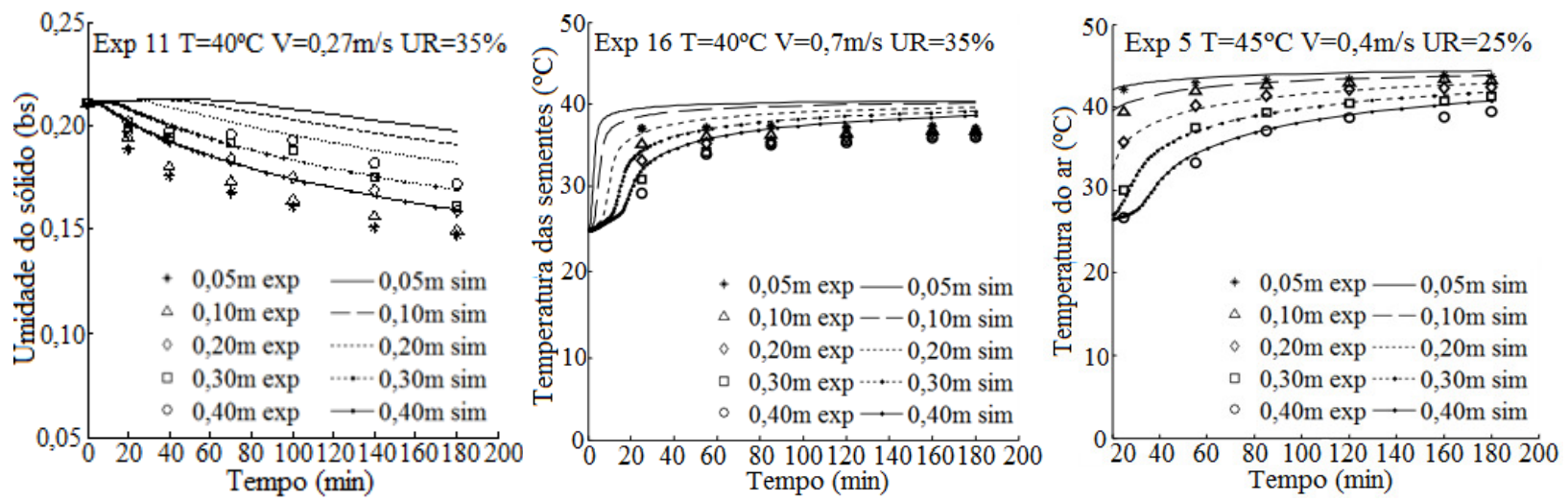

Figura 2. Perfis de umidade do sólido, temperatura das sementes de soja $\left({ }^{\circ} \mathrm{C}\right)$ e do ar de secagem, experimentais (exp) e simulados (sim) no decorrer do tempo para as posições na direção axial do leito, para o Exp. 2; Exp. 16 e Exp. 5, respectivamente.

Para todos os experimentos, houve variação da taxa de secagem das sementes nas diferentes posições axiais do leito, que não é uniforme. As diferenças entre as posições 0,05 e $0,40 \mathrm{~m}$ ficaram em torno de $0,30 \pm 0,05 \mathrm{pp} \cdot \mathrm{h}^{-1}$ para a maior parte dos experimentos. As maiores diferenças 


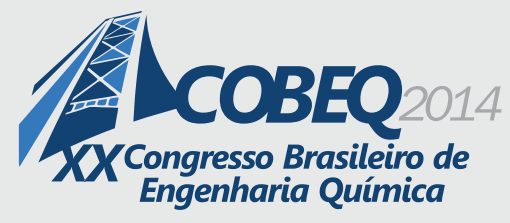

foram de 0,80, condição de baixa velocidade do ar de secagem (Figura 2 - Exp 11) e 0,49 pp.h ${ }^{-1}$, alta umidade relativa do ar $(49,1 \%)$.

A menor diferença entre as taxas de secagem nas diferentes posições, de $0,19 \mathrm{pp} \cdot \mathrm{h}^{-1}$, foi obtida na maior velocidade do ar de secagem $(1,12 \mathrm{~m} / \mathrm{s})$. Por conseguinte, também houve variação da umidade final das sementes nas diferentes posições axiais do leito o que permite assegurar que as sementes da camada superior, $0,40 \mathrm{~m}$, que estão em contato com o ar mais frio e mais úmido, apresentaram maiores valores de umidade (entre 13,6 e 17,2\% bs), evidenciando assim a heterogeneidade do produto. Este fato pode ser agravado com o aumento da espessura do leito, pois este tipo de secagem caracteriza-se por ocorrer em sucessivas camadas, ou seja, através de frentes de secagem (Peske e Villela; 2003). As menores umidades das sementes (entre 12,6 e $15,6 \%$ bs) e, consequentemente, as maiores temperaturas do ar e das sementes foram alcançadas nas posições mais próximas da entrada do ar no leito $(0,05$ e $0,10 \mathrm{~m})$, para cada condição experimental. De maneira geral, a taxa de secagem cresce com o aumento da temperatura e da velocidade, e com a diminuição da umidade relativa do ar de secagem, no entanto, esta combinação é desfavorável para a qualidade das sementes. Foi constatado que em apenas quatro dos dezesseis experimentos as sementes obtiveram a umidade final abaixo ou próximas de 13 a $14 \%$ (bs) nas diferentes posições axiais do leito, sendo que, dentre estes, apenas um apresentou condições menos desfavoráveis para a qualidade das sementes, com temperatura, velocidade e umidade relativa do ar de secagem de $40^{\circ} \mathrm{C}, 0,7 \mathrm{~m} / \mathrm{s}$ e $35 \%$, respectivamente, e umidades entre $13,3 \%$ (posição $0,05 \mathrm{~m}$ ) e 14,1\% (posição 0,40m).

Com relação a qualidade das sementes, a temperatura e a umidade relativa do ar de secagem foram as variáveis que influenciaram significativamente o índice de germinação e todas as variáveis estudadas influenciaram de alguma forma o índice de sementes sem fissuras das sementes de soja. Nas superfícies de respostas (Figura 3), pode-se visualizar que temperaturas e velocidades do ar mais baixas, assim como a umidade relativa mais alta favorecem a manutenção da qualidade das sementes, concordando com a literatura (Avelar et al., 2011; Krzyzanowski et al., 2006). O comportamento dos resultados exibidos na Figura 3 foi observado também nas demais posições do leito estudadas para cada índice apresentado.
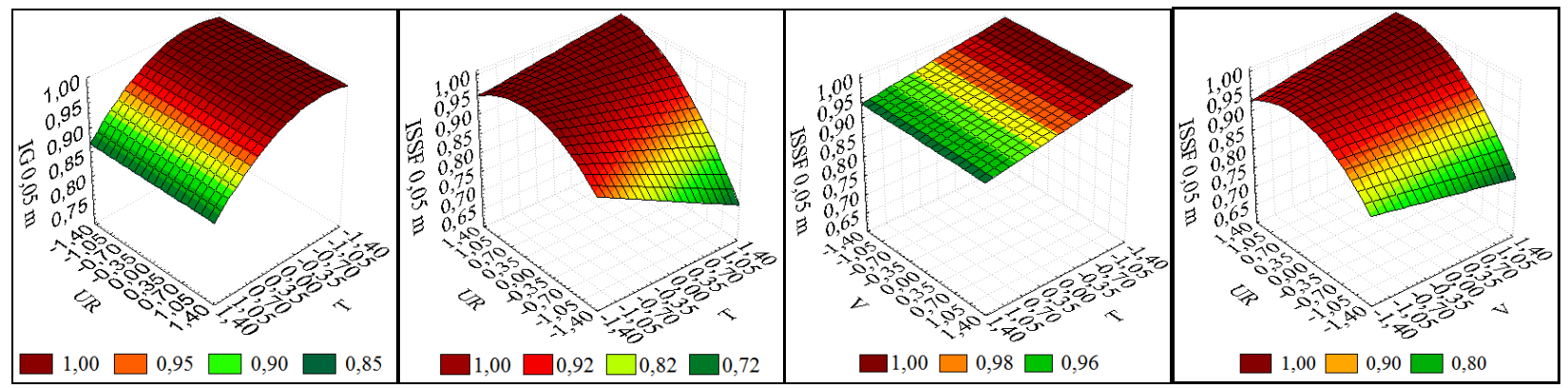

Figura 3 - Superfícies de resposta para a previsão dos índices de germinação (IG) e sementes sem fissuras (ISSF) das sementes de soja na posição axial do leito de 0,05 m. 


\section{CONCLUSÕES}

Os resultados do presente estudo demonstraram que o modelo de duas fases, com um conjunto adequado de equações constitutivas, previram com êxito a secagem em camada espessa das sementes de soja em um secador de leito fixo. Os resultados simulados tiveram uma boa concordância com os dados experimentais, com desvios médios variando de 1,4 a 5,7\%. A metodologia experimental desenvolvida se mostrou adequada para estudar a heterogeneidade do processo de secagem ao longo do leito, assim como a modelagem também previu com sucesso a heterogeneidade das variáveis de secagem. Verificou-se que apenas um experimento apresentou a combinação de umidade preconizada para o armazenamento das sementes $(13,3$ a $14,1 \%)$, e que temperaturas e velocidades do ar mais baixas, assim como a umidade relativa mais alta favorecem a manutenção da qualidade das sementes $\left(40^{\circ} \mathrm{C} ; 0,7 \mathrm{~m} / \mathrm{s}\right.$ e $\left.35 \%\right)$.

\section{AGRADECIMENTOS}

Os autores agradecem a Fundação de Amparo à Pesquisa do Estado de Minas Gerais (FAPEMIG) pelos recursos concedidos no Projeto de Participação Coletiva em Eventos Técnicos-Científicos (PCE-00082-14), o apoio financeiro recebido do CNPq e apoio financeiro e estrutural da Universidade Federal de Uberlândia.

\section{NOMENCLATURA}

Cp Calor específico a pressão constante $\left(\mathrm{J} \cdot \mathrm{kg}^{-1} \cdot{ }^{\circ} \mathrm{C}^{-1}\right)$

f Taxa de secagem por unidade de volume do leito $\left(\mathrm{kg} \cdot \mathrm{m}^{-3} \cdot \mathrm{s}^{-1}\right)$

G Vazão mássica $\left(\mathrm{kg} \cdot \mathrm{m}^{-2} \cdot \mathrm{s}^{-1}\right)$

hA Coeficiente volumétrico de transferência de calor sólido-fluido $\left(\mathrm{J} \cdot \mathrm{m}^{-3} \cdot \mathrm{s}^{-1} \cdot{ }^{\circ} \mathrm{C}^{-1}\right)$

$\mathrm{T} \quad$ Temperatura $\left({ }^{\circ} \mathrm{C}\right)$

t Tempo (s)

UR Umidade relativa (ad)

$\mathrm{V} \quad$ Velocidade superficial do fluido $\left(\mathrm{m} . \mathrm{s}^{-1}\right)$

y Coordenada da direção de escoamento do fluido

$\lambda \quad$ Calor latente de vaporização $\left(\mathrm{J} \mathrm{kg}^{-1}\right)$

$\rho \quad$ Densidade $\left(\mathrm{kg} . \mathrm{m}^{-3}\right)$

Subscritos

0 Condição inicial

f $\quad$ Fluido (ar)

s Sólido seco

v Vapor de água 


\section{REFERÊNCIAS}

AVELAR, S. A. G.; LEVIEN, A. M.; PESKE, S. T.; VILlELA, F. A.; BAUDET, L. Secagem estacionária de sementes de soja com ar desumidificado por resfriamento. Rev. Bras. Sementes, v. 33, n. 3, p. 454 - 462. 2011.

BARROZO, M.A.S.; SARTORI, D.J.M.; FREIRE, J.T.; ACHCAR, J.A. Discrimination of equilibrium moisture equations for soybean using nonlinearity measures. Dry. Technol., v. 14, n. 7, 1779-1794, 1996.

BARROZO, M.A.S.; MURATA, V.V.; COSTA, S.M. The drying of soybean seeds in countercurrent and concurrent moving bed dryers. Dry. Technol., v. 16, n. 9, 2033-2047, 1998.

BOX, M. J.; HUNTER, W. G.; HUNTER, J. S. Statistics for Experimenters, An Introduction to Design, Data Analysis, and Model Building. John Wiley and Sons, New York, 1978.

BROOKER, D. B.; BAKKER-ARKEMA, F. W.; HALL, C. W. Drying cereal grains. Westport, Connecticut. AVI Book, 1974.

LISBOA, M.H.; VITORINO, D.S.; DELAIBA, W.B.; FINZER, J.R.D.; BARROZO, M.A.S. A study of particle motion in rotary dryer. Braz. J. Chem. Eng., v. 24, n. 3, 265-374, 2007.

KRZYZANOWSKI, F.C.; WEST, S.H.; FRANCA NETO, J.B. Drying soybean seed using air ambient temperature at low relative humidity. Rev. Bras. Sementes, v.28, n.2, p.77-83. 2006.

LÓPEZ, A., PIQUÉ, M. T., ROMERO, A. Simulation of deep bed drying of hazelnuts. Dry. Technol., v. 16, n. 3, p. 651-665, 1998.

MYERS, R.H., MONTGOMERY, D.C. Response Surface Methodology: Process and Product Optimization Using Designed Experiments. New York: John Wiley and Sons, Inc., 2002.

OSBORN, G. S.; WHITE, G. M.; SULAIMAN, A. H.; WELTON, L. R. Predicting equilibrium moisture proportions of soybeans. T. ASAE, v. 32, n. 6, p. 2109-2113, 1989.

PESKE, S. T.; VILLELA F. Secagem de sementes. In: PESKE, S. T.; ROSENTHAL, M.; ROTA, G.R.M. Sementes: fundamentos científicos e tecnológicos. Pelotas: UFPel, 2003.

SOUZA, G. F. M. V. Secagem de sementes de soja em leito fixo: equilíbrio e cinética da sílica gel para controle de umidade, modelagem do processo e análise da qualidade das sementes. 2012. 157 f. Tese de Doutorado - Universidade Federal de Uberlândia.

WAKAO, N.; KAGUEI, S.; FUNAZKRI, T. Effect of fluid dispersion coefficients on particle-tofluid heat transfer coefficients in packed beds. Chem. Eng. Sci, v. 34, n. 3, 325 - 336, 1979. 